\title{
El estudio de la biblioteca digital académica en México mediante el uso de redes sociales
}

\author{
Georgina Araceli Torres Vargas *
}

Artículo recibido:

27 de septiembre de 2007.

Artículo aceptado:

29 de agosto de 2008.

\section{RESUMEN}

Se plantea el uso del análisis de redes sociales, como método útil para el estudio empírico de la biblioteca digital (BD) en México.

Palabras clave: Redes sociales, Biblioteca Digital académica.

* Centro Universitario de Investigaciones Bibliotecológicas de la UNAM, México. gatv@servidor.unam.mx

INVESTIGACIÓN BIBLIOTECOLÓGICA, Vol. 22, Núm. 46, septiembre/diciembre, 2008, México, ISSN: 0187-358X. pp. 41-57 
A study of the Academic Digital Library in Mexico through the use of social networks

Georgina Araceli Torres Vargas

The analysis of social networks is presented as a useful method for the empirical study of the Digital Library in México.

Keywords: Social networks; Academic Digital Library

\section{INTRODUCCIÓN}

L a Biblioteca Digital (BD), además de ser producto de las tecnologías de la información y la comunicación, es el resultado de la sociedad y su puesta en marcha está influida por los diversos actores sociales que participan en su implantación. A pesar del fuerte componente social de la $\mathrm{BD}$, hasta ahora ésta se ha estudiado más desde la perspectiva tecnológica, ya que se busca implementar equipos y programas para el desarrollo de servicios y procesos.

Ante esta situación el presente trabajo presenta una alternativa en el desarrollo de investigaciones de la biblioteca digital desde la perspectiva social, mediante el uso de la metodología de redes sociales.

El análisis que aquí se plantea realizar sobre la BD se da desde el punto de vista empírico; es decir, se pretende conocer cómo es el desarrollo de la BD mexicana en la actualidad, quiénes participan en su constitución y hacia dónde dirige su avance. Esto es posible en tanto que el estudio empírico se afianza en un modelo teórico de la BD que resulta de una investigación previamente realizada. Cabe resaltar que aquí no se pretende detallar el modelo; se parte directamente de la definición de BD y de los rasgos que la distinguen, puesto que la literatura al respecto ya se encuentra en proceso de ser publicada.

Desde el plano teórico, la BD debe contar con características muy particulares, pero en el plano real ¿qué tanto se acerca la biblioteca de un país determinado a este modelo ideal?

Se parte de la premisa de que cada país, dadas sus condiciones económicas, políticas, sociales, etcétera, propicia un desarrollo distinto en sus BD, y que lógicamente un país en desarrollo tendrá bibliotecas digitales muy diferentes de las de los países desarrollados. De ahí que sea necesario analizar lo que ocurre en cada país o región en materia de BD. 
Así la intención de este escrito es argumentar sobre la utilidad del análisis de redes sociales para el estudio de la BD en México, aunque podría ser aplicado a cualquier otra región. No se ofrecen datos sobre el estado que guarda actualmente la biblioteca digital porque éstos podrán conocerse luego de realizarse el estudio y mientras tanto no se puede decir nada acerca de los rasgos básicos de la $\mathrm{BD}$ académica mexicana ( por ejemplo acerca de los contenidos digitales, los medios para el acceso a la información, etcétera).

Puesto que a esta biblioteca puede analizársela desde diferentes ángulos, es necesario precisar que este artículo no aborda el uso de redes sociales para estudiar la producción de documentos digitales en la BD, ni el establecimiento de relaciones en cuanto a la cita de autores en el medio digital. Si bien a la fecha el uso de redes sociales en bibliotecología se ha afianzado con fuerza en el abordaje de aspectos bibliométricos, no es ése el caso del presente estudio.

\section{QUÉ ES LA BIBLIOTECA DIGITAL}

Para poder argumentar sobre el tema delimitado es necesario comenzar por plantear qué es una biblioteca digital:

La biblioteca digital es una biblioteca que se sustenta en un sistema de información documental en red, que ofrece a sus usuarios contenidos y servicios digitales, cuya información y medios de comunicación se encuentran en servidores distribuidos en diferentes latitudes del mundo. ${ }^{1}$

De esta definición se desprenden los siguientes rasgos básicos:

Sistema de información documental. Saorín identifica los sistemas de información documental (SID) de entre los diferentes tipos de sistemas de información, ${ }^{2}$ y señala que son aquellos que se encargan de la obtención, almacenamiento, recuperación y distribución de información sobre conocimiento registrado en un depósito o en una red de documentos. ${ }^{3}$

Pero no basta con caracterizar a la BD sólo como un sistema de información documental, sino que éste debe ampliarse hacia el concepto de red.

Red. Al hablar de biblioteca-red no se está hablando solamente de la biblioteca

1 Definición de la autora.

2 Se tipifican seis grupos de sistemas de información: sistemas de procesamiento de operaciones, de trabajo del conocimiento, de automatización de oficinas, de información para la administración, sistemas para el soporte de decisiones y sistemas de soporte gerencial.

3 Tomás Saorín Pérez, Los portales bibliotecarios, Madrid, Arco, 2004, pp. 135-136. 
conectada a redes, sino de la biblioteca como organización-red, en donde se adopta un papel de intercambio de información y procesos informativos con otras entidades con las que se relaciona y que se materializan muchas veces a través de las redes de comunicación. ${ }^{4}$

De esta forma, uno de los rasgos básicos de la BD es que es un sistema de información documental en red que descansa sobre tecnologías de la información y la comunicación (TIC). Es a partir del uso de las TIC como la $\mathrm{BD}$ se constituye en un conjunto de estructuras distribuidas que ofrece acceso a diversos repositorios.

Repositorio. En la actualidad el concepto de repositorio toma cada vez mayor relevancia gracias a que se concibe a la BD como un grupo de contenidos y servicios que se comparten con otras $\mathrm{BD}$.

El repositorio es un sistema de almacenamiento de contenidos digitales depositado por la institución, consorcio, país, o región, para proporcionarles servicios a sus comunidades en apoyo a la enseñanza y la investigación. Los repositorios deben ser considerados dentro del contexto del más amplio ambiente integrado de la información. Los servicios futuros dependerán de la interrelación de procesos entre repositorios, de las interfaces y de los demás componentes del ambiente de la información.

En realidad la BD distribuye la responsabilidad sobre contenidos y servicios, constituyéndose en un canal para nuevos mecanismos de coordinación y colaboración. ${ }^{5}$ No es posible definir la BD a partir de una unidad de información aislada; al acceder a la biblioteca se accede a recursos y sistemas de información documentales pertenecientes a redes de cooperación bibliotecaria en los que la biblioteca participa (además de los servicios suministrados por proveedores y distribuidores de información) y se accede así a productos o servicios cooperativos que son resultado del trabajo conjunto de las redes de bibliotecas. ${ }^{6}$

Los contenidos digitales organizados constituyen otro de los elementos característicos de la biblioteca digital; esto es, la información digital estructurada, a través de diversos tipos de publicación, y conformada por las colecciones ${ }^{7}$ de cada biblioteca con las que comparte información. Estos contenidos dispuestos en forma organizada a través de los repositorios

4 Rosalía Peña, Gestión digital de la información : de bits a bibliotecas digitales y la web, Madrid, Ra-Ma, 2002, p. 381.

5 Wendy Pradt Lougee, Diffuse libraries : emergent roles for the research library in the digital age, disponible en: http://www.clir.org/pubs/reports/pub108/html

6 Ibidem.

7 Tomando en cuenta la definición que da la Internacional Enciclopedia of Information and Library Science, en su segunda edición de 2003, se puede entender por colección a los documentos digitales seleccionados y acumulados que conforman una biblioteca digital. 
institucionales ${ }^{8}$ permitirán ofrecer servicios de información digitales mediante la colaboración con otras bibliotecas tanto para el préstamo de los mismos como para su administración. Hablamos así de servicios en colaboración.

Estos son los rasgos básicos que se observan en el modelo teórico de lo que debe ser una biblioteca digital.

Pero si hablamos de lo que se considera una BD académica, hay que acotar que la tipología de la BD suele estar conforme con la comunidad a la que sirve, a sus objetivos, actividades, características, etcétera. Tales características determinan si se trata de una biblioteca digital pública, escolar, nacional o académica. En consecuencia La BD académica será aquella biblioteca que forma parte de una universidad o institución de educación superior, administrada para satisfacer las necesidades de su comunidad.

A pesar de que la biblioteca académica es considerada como sinónimo de biblioteca universitaria, ${ }^{9}$ en años recientes se ha optado por el uso del término biblioteca académica, porque se le considera una alternativa de estructura organizacional, frente a los cambios tecnológicos que han afectado algunas de las operaciones bibliotecarias. ${ }^{10}$

Si bien la biblioteca académica sustenta las labores de docencia e investigación de una universidad, hay que decir que al estudiar la BD académica cabe tener en mente que ésta debe atender de manera particular los aspectos docente y de investigación de la institución a la que pertenece, ya que cada una de estas vertientes muestra requerimientos distintos al momento de diseñar servicios, desarrollar colecciones digitales, etcétera.

Tras estas precisiones conceptuales podemos ver ahora el planteamiento del uso de redes sociales en el estudio de la BD académica en México.

\section{Algunas áreas en las que se hace uso de Redes sociales}

El tema de redes sociales ha sido desarrollado desde distintas perspectivas. Algunas de las áreas en donde se han utilizado las redes son: terapia familiar

8 Los repositorios institucionales se aplican sobre todo al contexto de las labores de docencia e investigación. Un repositorio institucional puede servir para la publicación de artículos de investigación, tesis, reportes, conferencias, etcétera. Cfr. Richard Jones, The institutional repository, Oxford, Chandos, 2006, p. 11.

9 Joan M. Reitz, Online Dictionary for Library and Information Science, disponible en: http:// lu.com/olis/

10 Stephen E. Atkins, The academic library in the American university, Madison, Wisconsin, Parallel, 2003, p. 163. 
(donde se desarrollaron propuestas de abordaje barrial y comunitario); pero también se han hecho aportes en sociología, donde se relacionan los procesos de marginalización generados por el desempleo, la patología económica y la social, la pérdida de identidad, de pertenencia y de seguridad. También el análisis institucional y de grupos ha sido útil para examinar las prácticas comunitarias en salud mental. ${ }^{11}$

En el caso de la bibliotecología, como ya se señaló, el estudio de redes sociales se ha usado con frecuencia en los estudios bibliométricos, en donde hay numerosos estudios que se podrían citar. Sin intención de ser exhaustivos, se pueden ver los siguientes ejemplos:

Buzydlowski, Jan, "Co-Cited Author Maps as Interfaces to Digital Libraries, Designing Pathfinder Networks in the Humanities", en Proceedings of the International Conference on Information Visualisation, Washington, DC, IEEE Computer Society, 2000, p. 25.

Chen, C., "Visualising semantic spaces and author co-citation networks in digital libraries", en Information Processing and Management: an International Journal, Volume 35, No., 3, (May., 1999), pp. 401-420.

Braz de Oliveira e Silva, Antonio, "Estudo da rede de co-autoria e da interdisciplinaridade nao producao científica com base nos métodos de análise de redes sociais : avaliacao do caso do programa de pósgraduacaoem ciencia da informacao-PPGCI/UFMG, pp. 179-194, en Encontros Bibli, Núm. Especial, 2006, disponible en: http://www. encontros-bibli.ufsc.br/especial.html

Molina, José Luis, "Redes de publicaciones científicas: un análisis de la estructura de coautorías", en REDES : Revista Hispana para el Análisis de Redes Sociales, Vol.1, No. 3, (ene., 2002), disponible en : http:// revista-redes.rediris.es/html-vol1/vol1_3.htm

Tomaél, Maria Inés, "Redes sociais : posicoes dos atores no fluxo da informacao”, en Encontros bibli, Núm. Especial, 1er semestre de 2006, disponible en: http://www.encontros-bibli.ufsc.br/especial.html

White, Howard D., "Pathfinder networks and author cocitation analysis: a remapping of paradigmatic information scientists" en Journal of the American Society for Information Science and Technology, Vol.54, Núm..5, (Mar.,2003), pp.423-434.

11 Elina Nora Dabas, Red de redes : las prácticas de la intervención en redes sociales, Buenos Aires, Paidós, 1993, pp. 15-16. 
Esta tendencia obedece sobre todo a que como lo señala Vivas, ${ }^{12}$ las redes sociales son útiles como instrumento adecuado para un abordaje complementario a las técnicas bibliométricas tradicionales, ya que facilita el análisis estructural de las relaciones sociales. El mismo autor asevera que para quienes están familiarizados con los trabajos sobre colaboración científica, el estudio de las relaciones entre autores no constituye una novedad. ${ }^{13}$

Esta familiaridad ha hecho que al hablar de redes sociales en el área de la bibliotecología y de los estudios de la información, se piense de manera casi inmediata en estudios de corte bibliométrico y, por consiguiente, en el uso de herramientas estadísticas que tan ligadas están a estos estudios, aun cuando no son las únicas herramientas que existen en el análisis de redes sociales.

Pero además de este ámbito las redes sociales pueden ser útiles en el estudio de otros aspectos bibliotecológicos. El que aquí se menciona es el que corresponde a la BD y para plantearlo es necesario dejar claros los elementos que se recogen de las redes sociales. ${ }^{14}$

\section{Elementos De LAS REDES SOCIALES, PARA EL ESTUdio DE LA BD ACADÉMICA EN MÉXICO}

En el ámbito de la sociología estructural se establece que todas las estructuras sociales pueden ser conceptualizadas como redes, en donde los nodos representan a los actores y las áreas que conectan a los nodos representan relaciones entre actores. ${ }^{15}$

De acuerdo con Lomi, ${ }^{16}$ el significado del término red es: ...mecanismo sistemático de representación y codificación de las interacciones que hay entre las unidades de observación.

Además las unidades básicas de observación en una red social son los actores sociales (personas u entidades) que se vinculan entre sí. ${ }^{17}$ En estricto

12 Jorge Ricardo Vivas, "Análisis de redes sociales a la información sociobibliométrica", PsicoUSF, Vol. 9, Núm. 1, ( jun. 2004), - p.78.

13 Ibid., p. 81.

14 En el entendido de que el método de análisis de redes sociales ofrece un gran abanico de posibilidades en el presente artículo se muestran aquellas que se observan como las más adecuadas, de acuerdo con las características del estudio.

15 Cfr. Peter Hedstrom, "Introduction to the special issue on social network analysis", en Acta Sociológica, Vol 37, Núm. 4, (1994), p. 327.

16 Alessandro Lomi, "L'etude relationelle de l'organisation sur différents plans d'analyse. Introduction”, pp. 23-57, en L'analyse relationnelle des organizations : reflexions theoriques et experiences empiriques, Alessandro Lomi (sous la direction de) ; traduit del l'italien par Luciana T. Soliman, Paris, L'Harmattan, 1999, p. 29.

17 Análisis de redes : aplicaciones en ciencias sociales, Jorge Gil Mendieta, Samuel Schmidt, eds., México, IIMAS, 2002, p. 2. 
sentido sociológico, los grupos, asociaciones, compañías, autoridades, instituciones, estados u otras colectividades sociales son considerados como actores si los procesos, eventos u otros resultados son atribuidos a la colectividad y no son causa de subunidades o miembros individuales. ${ }^{18}$ En el nivel más bajo de agregación, los actores individuales pueden ser estudiados en la medida en que se involucren en relaciones interpersonales, ${ }^{19}$ pero en general desde la perspectiva del análisis de redes, la estructura social es un tejido permanente de lazos entre posiciones sociales, más que entre individuos. ${ }^{20}$

En relación con esto, Mancur Olson ${ }^{21}$ asevera que las organizaciones que producen "bienes colectivos" no pueden operar adecuadamente en relación con motivaciones, valores y objetivos en un nivel individual, sino que los individuos deben identificarse fuertemente con la organización. ${ }^{22}$ De allí que cuando se habla de redes sociales se hace referencia a organizaciones, que son el espacio en el que se conforman las redes sociales.

El individuo que está inserto en una organización guarda un comportamiento basado en intercambios directos o indirectos con otros individuos, pero la organización capacita y persuade a sus miembros a responder a partir de los requerimientos de su puesto; sus sentimientos internos y su comportamiento externo se basan en el entrenamiento organizacional. ${ }^{23}$ Por esta razón el comportamiento en las organizaciones tiene una base organizacional, más que individual.

Hoy se considera que las organizaciones tienen una existencia en sí mismas, más allá del comportamiento y desempeño de los individuos dentro de ellas. Para Hall, una muestra de ello es el hecho de que las organizaciones perduren en el tiempo y reemplacen a sus miembros, pues esto sugiere que no son dependientes de los individuos particulares. Como ejemplo, asevera que “[...]las universidades viven más que las generaciones de estudiantes y

18 Hans Geser, Organizations as a social actors, en Sociology in Switzerland: online publications, disponible en http://socio.ch/arbeit/t_hgeser5.htm

19 Un ejemplo de la utilidad de las redes sociales en el estudio de relaciones interpersonales es el estudio de Schmidt y Gil Mendieta, quienes analizan la red de poder mexicana a través de las relaciones entre personalidades de la política, ( $c f r$. Schmidt, Samuel, "La red de poder mexicana”, pp. 95-155, en Análisis de redes aplicaciones en ciencias sociales., op.cit.).

20 Análisis de redes : aplicaciones en ciencias sociales., op. cit., pp. 7, 9.

21 Cfr. Mancar Olson, The logic of collective action, Cambridge : Harvard University Press, 1965.

22 Hall señala que "Una organización es una colectividad con una frontera relativamente identificable, un orden normativo, niveles de autoridad, sistemas de comunicaciones y sistemas de coordinación de membresías; esta colectividad existe de manera continua en un ambiente y se involucra en actividades que se relacionan por lo general con un conjunto de metas; las actividades tienen resultados para los miembros de la organización, la organización misma y la sociedad". (Hall, Richard, Organizaciones : estructuras, procesos y resultados, México, Prentice Hall, 1996, p. 33).

23 Richard Hall, op. cit., p. 34. 
maestros que pasan por sus puertas”. ${ }^{24}$ En este sentido es válido que el análisis de redes sociales se centre en el estudio de los grupos de actores (no de los individuos) y de su entorno.

Lo anterior se refuerza con lo señalado por Kilduff, ${ }^{25}$ quien asevera que la emergencia de una crítica a las redes mediante el aprovechamiento de las teorías organizacionales, contribuye en la investigación en áreas cercanas a esta perspectiva; por ejemplo -añade-- los estudiosos se han integrado a las redes sociales para el estudio de la formación de estrategias y alianzas mediante varias formas de relaciones interorganizacionales.

Para esquematizar las relaciones que se dan entre actores, son útiles las representaciones visuales. Éstas ayudan al investigador a comprender propiedades de la red que de otra forma serían difíciles de observar; por eso un aspecto básico en el análisis de redes sociales son los sociogramas, en donde se representan las relaciones sociales. Las conexiones que se representan gráficamente son la estructura relacional de un grupo social ${ }^{26}$

Pero un sociograma no puede elaborarse tan solo a partir del uso de alguno de los programas de cómputo que existen; los sociogramas se elaboran a partir de los datos que se obtienen mediante el uso de las técnicas de investigación que se delimiten (ya sean encuestas, entrevistas, etcétera).

Gráficamente un sociograma puede mostrarse de la siguiente manera:

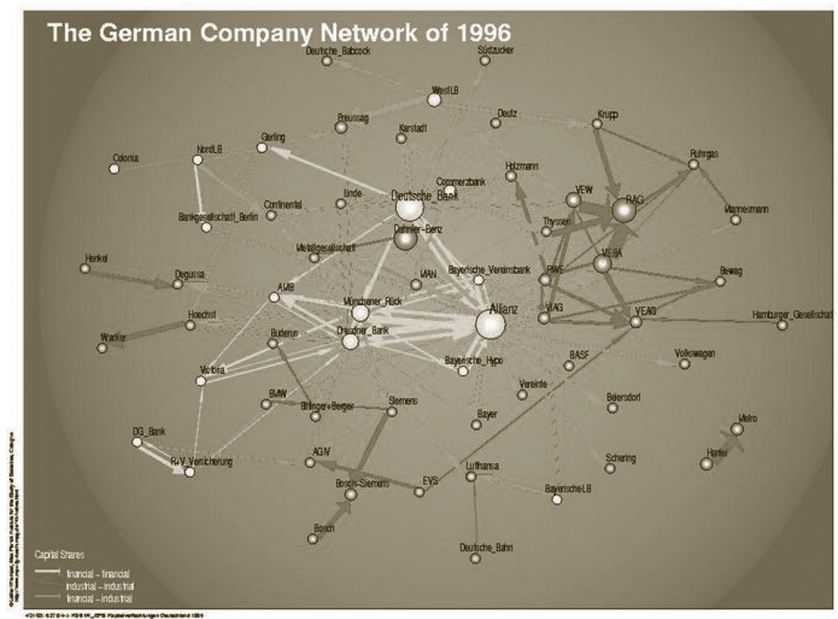

La red de compañías alemanas en 1996. Fuente: Galería de NetVis ${ }^{27}$.

24 Ibid., p. 36.

25 Martin Kilduff, Social networks and organizations, London: Sage, 2003, p. 63.

26 Hannah Knox, Social networks and the study of relations : networks as method, metaphor and form, pp. 113-140, en Economy and society, Vol. 35, Núm. 1, (feb., 2006), p. 117.

27 Disponible en: http://www.mpi-fg-koeln.mpg.de/ lk/netvis/substanz.html 
Es preciso señalar que el uso metafórico de la imagen de una red tal y como se muestra en los sociogramas resulta atractivo, pero la imagen de una red sólo puede ser útil científicamente cuando se la precisa como un modelo empírico que parte de un modelo teórico.

Es necesario hacer un paréntesis para ahondar sobre esta última aseveración: Las redes sociales ayudan a mostrar lo que Searle llama hechos sociales ${ }^{28}$ y que se refieren a cualquier hecho que involucra una intencionalidad colectiva; por ejemplo, el que dos personas vayan a trabajar juntas es un hecho social. Una clase especial dentro de éstos son hechos institucionales.

Estos hechos no pueden verse a simple vista sino que es necesario acercarse a ellos a través del conocimiento de las prácticas que se llevan a cabo al interior de la institución, y un medio para lograr esto son las redes sociales. Para algunos autores el método de redes significa el mapeo que hace comprensible lo "real". ${ }^{29}$

En relación con esto, Lomi asevera que una de las labores fundamentales del estudio de redes consiste en reconstruir toda la estructura de relaciones. Y que el análisis de estas relaciones le confiere un carácter empírico al estudio, ya que tales relaciones se elaboran de forma específica para representar y comprender los fenómenos que subyacen en la organización. ${ }^{30}$

Pero aunque a partir de un estudio de redes sociales es posible reflejar el modelo empírico de la BD académica, es necesario confrontar este modelo con uno teórico, pues sin un referente teórico no hay posibilidad de interpretación sobre lo que sucede en la organización y todo resultaría una simple descripción.

El modelo teórico a su vez podrá enriquecerse con los elementos que se encuentren en la situación real de la BD; lo que convierte en un continuo el conocimiento sobre este tipo de biblioteca. No es suficiente estudiar a la BD desde la teoría sino que se requiere un acercamiento a la realidad para que ni la teoría ni la práctica transiten por caminos separados. Esta necesidad de relación se hace más patente en un tema que, como el de la BD, se constituye en gran medida por aspectos que se derivan del uso de las tecnologías de la información y la comunicación, donde se corre el peligro de dejarse llevar más por la práctica que por el análisis teórico de lo que sucede y de lo que debería suceder. 


\section{Planteamiento para el estudio de la bD aCAdémica mexicana}

MEDIANTE LAS REDES SOCIALES

En un plano general el estudio de la BD académica desde el enfoque de redes sociales puede contribuir al conocimiento de los actores, sus roles y las aportaciones de cada uno de los participantes en la red. Pero estos actores y roles están influenciados por el ambiente en el que se encuentran.

Por ejemplo, para visualizar los roles es necesario correlacionar a los actores con las instituciones a las que pertenecen, ya que solo a partir de esta relación es factible que la institución manifieste su experiencia, dado que la institución es un ensamblaje de acciones "programadas" (como una puesta en escena) cuya realización depende de los papeles que determinan los roles de cada actor. ${ }^{31}$

En el caso que nos ocupa es menester hablar de la biblioteca digital académica como parte de una institución: la universidad. Ésta constituye uno de los elementos fundamentales dentro de un estudio de la BD académica desde la perspectiva de redes sociales.

Hay que considerar que las estructuras organizacionales de las universidades son muy complejas, ambiguas y anárquicas. Además de que al interior de la universidad coexisten diversos objetivos, problemáticas, estrategias y conflictos. ${ }^{32}$ La universidad, al igual que los demás espacios de la sociedad, recibe múltiples influencias, a la vez que interactúa con varios factores económicos, políticos, sociales y culturales. Es por eso que uno de los aspectos que es necesario analizar para poder estudiar a la BD académica desde el enfoque de redes sociales es la universidad de la cual depende, misma que a su vez está condicionada por un contexto nacional.

Podemos aseverar que en México se desarrolla una universidad cuyas características la diferencian de la universidad británica, de la estadunidense o de la universidad de cualquier otra latitud. Si bien en opinión de algunos ya no es posible hablar de universidades geográficamente diferenciadas, sobre todo por efectos de la globalización, en palabras de Ibarra Colado

"[...]se ha empezado a consolidar una nueva división internacional del trabajo universitario [...], existen diferencias entre regiones, países y localidades, alejándonos

31 Meter L. Berger, The social construction of reality, London, Penguin, 1967, p. 92.

32 Organizaciones como la Universidad, pueden entenderse como organizaciones duales; es decir, organizaciones que tienen una compleja relación entre su dinámica interna y sus relaciones con el contexto externo, en otras palabras, con el espacio social específico en el que se ubican. Estas organizaciones son formales y complejas, a la vez que operan en formas ambiguas y erráticas. Y, estas formas de operar, parecieran, en primera instancia, bloquear toda iniciativa de cambio. 
con ello de la grosera interpretación de las mega-tendencias globales, usualmente presentadas como realidades inescapables antes las cuales no hay alternativa". ${ }^{33}$

Así, lo que se observa es la posibilidad de analizar a la BD a partir de la relación directa que tiene con la universidad mexicana y desprender así sus características y problemáticas.

Además del factor institucional en el que está inmersa la BD académica mexicana, es necesario conocer a sus actores sociales ${ }^{34}$ y las relaciones que se dan entre ellos. Pero ¿como identificar a tales actores? De acuerdo con Laumann, ${ }^{35}$ la investigación tiene que ser definida a partir del criterio de un análisis nominalista o de un criterio realista. ${ }^{36}$ Este último se aplica a partir de la percepción de los actores en una unidad social preexistente. Como señala Molina, ${ }^{37}$ podemos estudiar un club deportivo, un grupo de jóvenes, un barrio, etcétera, siempre que los actores consideren que existe una entidad. En el caso que nos ocupa la entidad es la BD académica en cuestión y a partir del análisis de datos en torno de ésta, es como podrán identificarse a los actores que intervienen en su conformación.

En este caso la recolección de datos es una cuestión esencial desde el momento en que trabajamos con redes sociales. Los métodos de recolección de datos que se utilizan en el análisis de redes sociales son los cuestionarios, las entrevistas en profundidad, la observación y los registros documentales. ${ }^{38}$ Con ayuda de estos métodos es posible reconocer a los actores involucrados.

Ahora bien, en cuanto a las relaciones que se establecen entre los actores es preciso señalar que éstas se pueden analizar desde alguna de las siguientes perspectivas: los lazos como medios de flujo de recursos entre las organizaciones, los lazos como vínculos entre grupos de actores, y los lazos como indicadores concretos de patrones abstractos de relaciones en las que los actores están inmersos.

Una red social se interpreta básicamente por la información relacional que ésta contiene, a diferencia de los modelos que se han trabajado por tradición

33 Eduardo Ibarra Colado, "La 'nueva universidad' en México : transformaciones recientes y perspectivas", pp. 75-105, en Revista Mexicana de Investigación Educativa, Vol. 7, Núm. 14, (ene.-abr., 2002), p. 78.

34 Tal y como ya se mencionó en páginas anteriores, en este caso los actores sociales deben ser considerados como colectividades y no como individuos.

35 Edward O. Laumann, "The boundary specification problem in network analysis", en Ronald Burt y Michael J, eds., Applied network analysis, Beverly Hills, Sage, 1983, p. 19.

36 En una aproximación inicial podría suponerse cuáles son los actores de la BD académica en México, haciendo uso del criterio nominalista que señala Laumann. Pero en el caso del diagnóstico de la BD que se persigue, se prefiere iniciar el estudio sin algún supuesto que pueda guiar al estudio hacia lo que creemos que sucede, en un intento por ser lo más objetivo posible. Se prefiere por tanto el criterio de análisis realista.

37 José Luis Molina, El análisis de redes sociales : una introducción, Barcelona, Bellaterra, 2001, p. 65. 
en las ciencias sociales, en donde los atributos de las unidades independientes son el tema central del análisis. Es así como se tiene una herramienta para estudiar el estado que guarda la BD académica: frente a un modelo teórico de sí misma.

Independientemente del tópico o la disciplina, los fenómenos sociales son concebidos como un sistema de unidades conectadas. ${ }^{39}$ De acuerdo con Freeman. ${ }^{40}$ Una red social consiste, en esencia, de dos elementos: una población de actores y por lo menos una relación que sea cuantificable y definida para cada par de actores.

Así a partir de un estudio de corte empírico de la BD académica en México basado en el análisis de redes sociales, será posible identificar entonces otros aspectos de esta biblioteca que a simple vista no se observan y que se refieren tanto a los actores que la determinan como a la forma en que se relacionan estos actores. El mapeo de tales relaciones será importante para poder determinar en qué estado de desarrollo se encuentran éstas a partir de los problemas y fortalezas que se reflejan en la red social, siempre frente a un modelo teórico previamente acotado y bajo un contexto nacional.

Es importante que la recolección de datos se plantée sobre la base de una muestra representativa de bibliotecas digitales académicas.

Para determinar la muestra se reitera la necesidad de contar con un referente teórico de lo que se quiere analizar a la luz de las redes sociales, pues para identificar a las bibliotecas digitales académicas en México es necesario establecer antes los rasgos que caracterizan a una BD, independientemente de su tipo.

Para iniciar el estudio de BD académicas en México se cuenta con un modelo teórico de la BD, del cual se señalaron ya los elementos básicos.

Este modelo, además de establecer una definición de lo que es la BD, señala las partes que le dan estructura a esta biblioteca y las características que debe tener.

La colaboración entre bibliotecas digitales (a diversos niveles) se establece como una condición, y esto ha dado pie a la creación de consorcios de bibliotecas como forma de organización bibliotecaria. ${ }^{41}$ Esta es una condición básica para considerar que una biblioteca es digital, por lo que las BD más desarrolladas mostrarán este rasgo.

39 Ibid., p. 10.

40 L. C. Freeman, "Social networks and the structure experiment", en Research methods in social networks analysis, Fairfax, V, Mason University Press, 1989.

41 Un consorcio puede definirse como una "[...] sociedad formada por un grupo de bibliotecas, generalmente restringidas a zonas geográficas, a una clase de bibliotecas o al interés por una materia, que se constituye para desarrollar y compartir los recursos de todos los miembros y para ello mejorar los servicios bibliotecarios y los recursos de que disponen sus respectivos grupos de usuarios". Cfr. Antonia Ferrer Sapena, Guía metodológica para la implantación de una biblioteca digital universitaria, Madrid, TREA, 2005, p. 146. 
Por consiguiente para delimitar una muestra de bibliotecas digitales académicas en México será indispensable que guarden la característica de estar constituidas en consorcios.

Para tener una primera aproximación a las BD académicas en México, hay que referirnos a la cifra que menciona Margarita Juárez con respecto de lo que ella llama proyectos colectivos, entre los que se encontrarían grupos de bibliotecas bajo algún consorcio. La autora menciona que en México encontramos más número de proyectos individuales (89\%) que colectivos $(11 \%){ }^{42}$

Como puede verse, las características que teóricamente determinan a una $\mathrm{BD}$, serán las que guíen en diversas fases el análisis de redes.

El mapeo de las relaciones entre los actores que intervienen en la biblioteca digital académica en México y la visualización gráfica de las mismas, serán el resultado tanto del uso del método de redes sociales, como de la continua interrelación entre el método y el modelo teórico referente. El estudio permitirá retroalimentar el modelo y en su caso corregirlo, añadirlo, etcétera, y el modelo a su vez, ayudará como hilo conductor para el análisis de redes.

\section{Conclusiones}

A partir de los planteamientos que se detallan en este artículo se concluye que:

- Aunque el tema de redes sociales ha sido desarrollado desde áreas como la antropología y la psicología, entre otras, en la bibliotecología es escaso el número de estudios que han hecho uso de las redes sociales y los pocos trabajos que hay se ubican en el área de la bibliometría. No existe ningún documento que analice a la biblioteca digital con base en esta metodología.

- Las redes sociales se integran por los actores sociales y los lazos que se establecen entre los actores. Desde la perspectiva del análisis de redes es pertinente analizar a la BD como organización, más que a través de los individuos que intervienen en ella.

- Para esquematizar las relaciones que se dan entre los actores son útiles las representaciones visuales, materializadas en sociogramas creados con ayuda de programas de cómputo adecuados para ello. Los

42 Margarita Juárez Álvarez, "El panorama actual de las bibliotecas digitales en México”, pp. 193-226, en Jornadas Mexicanas de Biblioteconomía (37: 2007: León, Guanajuato) Memorias, México, AMBAC, 2007, disponible en: http://www.ambac.org.mx/publicaciones/memorias_ XXXVIII_jornadas.pdf 
sociogramas auxilian al investigador a interpretar y comprender las propiedades de la red, pero estas representaciones deben considerarse como un modelo empírico. Su interpretación se complementa con un modelo teórico del objeto de estudio. En este caso con un modelo teórico de la $\mathrm{BD}$.

- Para analizar los roles de los actores y sus relaciones también es necesario correlacionar a los actores con las instituciones a las que pertenecen. En el caso que nos ocupa hay que tomar en cuenta a la universidad como institución que cobija a la BD académica. Esta universidad está a su vez condicionada por un contexto nacional, que también es necesario considerar. Por eso se habla de la BD académica en México.

- Para conocer a los actores sociales se prefiere el criterio de análisis realista, ya que éste permite identificarlos sin alguna información inicial y ayuda a ser más objetivos.

- Bajo el criterio de análisis realista habrá que hacer una recolección de datos que permita conocer a los actores sociales de la BD académica en México y las relaciones que existen entre ellos. Es importante que la recolección de datos se plantee sobre la base de una muestra representativa de bibliotecas digitales académicas, para lo cual se cuenta con un modelo teórico de la BD que ayudará en esta fase. Una de las condiciones que se establecen a partir de este modelo es que la biblioteca académica esté constituida en consorcio para poder considerarla como biblioteca digital (además de otros elementos que se señalan en este escrito), ya que las BD más desarrolladas muestran este rasgo.

- En definitiva el uso del análisis de redes sociales para el estudio de la biblioteca digital académica en México arrojará datos de carácter empírico que nos permitirán conocer a los actores sociales que están interviniendo en su constitución, y las relaciones que se dan entre ellos, a través del trazo de un mapeo de lazos. Este estudio empírico, en conjunción con el modelo teórico de la BD, ayudará a conocer el curso que sigue la BD en nuestro país. Esto contribuirá a nuestra comprensión de su desarrollo, sus debilidades y los rasgos más sólidos que se pueden rescatar de ella. Todo ello para seguir trabajando a favor de la construcción de una BD académica idónea para el contexto mexicano. 


\section{BiBLIOGRAFÍA}

Análisis de redes : aplicaciones en ciencias sociales / Jorge Gil Mendieta, Samuel Schmidt, eds, México, IIMAS, 2002.

Atkins, Stephen E., The academic library in the American university, Madison, Wisconsin, Parallel, 2003.

Berger, Meter L., The social construction of reality, London, Penguin, 1967.

Braz de Oliveira e Silva, Antonio, "Estudo da rede de co-autoria e da interdisciplinaridade nao producao científica com base nos métodos de análise de redes sociais : avaliacao do caso do programa de pós-graduacaoem ciencia da informacao-PPGCI/UFMG”, pp. 179194, en Encontros Bibli, Núm. Especial, 2006, disponible en: http://www.encontros-bibli.ufsc.br/especial.html

Fabio, Severino di, "La Social Network Analisys sui sistemi bibliotecari: il caso civico romano", pp. 439-449, en Bollettino AIB, Vol. 45, 45 Núm. 4, (2005).

Ferrer Sapena, Antonia, Guía metodológica para la implantación de una biblioteca digital universitaria, Madrid, TREA, 2005.

Freeman, L. C., "Social networks and the structure experiment", en Research methods in social networks analysis. Fairfax, V, Mason University Press, 1989.

Galería de NetVis, disponible en: http://www.mpi-fg-koeln.mpg. $\mathrm{de} / \sim \mathrm{k} /$ netvis/substanz.html

Geser, Hans, "Organizations as a social actors", en Sociology in Switzerland: online publications, disponible en http://socio.ch/arbeit/t_ hgeser5.htm

Hall, Richard, Organizaciones : estructuras, procesos y resultados, México, Prentice Hall, 1996.

Hedstrom, Peter, Introduction to the special issue on social network analysis, pp. 327-328, en Acta Sociológica, Vol. 37, Núm. 4, (1994).

Ibarra Colado, Eduardo, "La 'nueva universidad' en México : transformaciones recientes y perspectivas”, pp. 75-105, en Revista Mexicana de Investigación Educativa, Vol. 7, Núm. 14, (ene.-abr., 2002).

International encyclopedia of information and library science, edited by John Feather and Paul Sturges. London, Routledge, 2003, xxxii, 688 p. I

Juárez Álvarez, Margarita, "El panorama actual de las bibliotecas digitales en México”, pp. 193-226, en Jornadas Mexicanas de Biblioteconomia (37: 2007: León, Guanajuato) Memorias, México, AMBAC, 2007, disponible en: http://www.ambac.org.mx/publicaciones/ memorias_XXXVIII_jornadas.pdf

Knox, Hannah, "Social networks and the study of relations : networks as method, metaphor and form", pp. 113-140, en Economy and society, Vol. 35, Núm. 1, (feb., 2006). 
Laumann, Edward O., "The boundary specification problem in network analysis", en Ronald Burt y Michael J, eds., Applied network analysis, Beverly Hills, Sage, 1983.

Lomi, Alessandro, "L'etude relationelle de l'organisation sur différents plans d'analyse. Introduction”, pp. 23- 57, en L' analyse relationnelle des organizations: reflexions theoriques et experiences empiriques, Alessandro Lomi (sous la direction de), traduit del l'italien par Luciana T. Soliman, Paris, L'Harmattan, 1999.

Molina, José Luis, El análisis de redes sociales : una introducción, Barcelona, Bellaterra, 2001.

Nora Dabas, Elina, Red de redes : las prácticas de la intervención en redes sociales, Buenos Aires, Paidós, 1993.

Olson, Mancar, The logic of collective action, Cambridge, Harvard University Press, 1965.

Reitz, Joan M., Online Dictionary for Library and Information Science, disponible en: http://lu.com/olis/

Searle, John R., The construction of social reality, New York, Free Press, 1995.

Tomaél, Maria Inés, "Redes sociais : posicoes dos atores no fluxo da informacao", en Encontros bibli, Núm. Especial, 1er semestre de 2006, disponible en: http://www.encontros-bibli.ufsc.br/especial. html 